\title{
Risk of dementia in patients hospitalised with type 1 and type 2 diabetes in England, 1998-2011: a retrospective national record linkage cohort study
}

\author{
Kate Smolina • Clare J. Wotton • Michael J. Goldacre
}

Received: 23 October 2014 / Accepted: 15 January 2015 / Published online: 12 February 2015

(C) Springer-Verlag Berlin Heidelberg 2015

\begin{abstract}
Aims/hypothesis Type 2 diabetes increases the risk of subsequent dementia. Our objective was to determine whether a similar risk of subsequent dementia is associated with type 1 diabetes in a large defined population.

Methods This retrospective cohort study examined national administrative record-linked statistical data on hospital care and mortality in England, 1998-2011. Cohorts of people admitted to hospital when aged 30 or over were constructed: 343,062 people with type 1 diabetes; $1,855,141$ people with type 2 diabetes; and a reference cohort. Results were expressed as rate ratios (RR) comparing each diabetes cohort with the control cohort.

Results The overall RR for dementia in people admitted to hospital with type 1 diabetes was 1.65 (95\% CI 1.61, 1.68), and for people admitted to hospital with type 2 diabetes was $1.37(1.35,1.38)$. Young age at admission for diabetes appeared to confer a greater rate of subsequent dementia; the RR for dementia in people admitted to hospital with type 1 diabetes aged $30-39$ years was $7.10(4.65,10.6)$, which reduced to $4.40(3.55,5.40)$ in those aged $40-49$ at admission, and further reduced with increasing age to $1.16(1.11,1.20)$ in those aged 80 or over at admission. A similar pattern was seen with type 2 diabetes.
\end{abstract}

Electronic supplementary material The online version of this article (doi:10.1007/s00125-015-3515-x) contains peer-reviewed but unedited supplementary material, which is available to authorised users.

K. Smolina

Centre for Health Services and Policy Research, School of

Population and Public Health, University of British Columbia,

Vancouver, BC, Canada

K. Smolina $\cdot$ C. J. Wotton $\cdot$ M. J. Goldacre $(\bowtie)$

Unit of Health-Care Epidemiology, Nuffield Department of

Population Health, University of Oxford, Old Road Campus,

Old Road, Oxford OX3 7LF, UK

e-mail: michael.goldacre@dph.ox.ac.uk
Conclusions/interpretation Type 1 diabetes, as well as type 2 diabetes, may be associated with an elevated risk of subsequent dementia. The risk of dementia varies with age at admission to hospital with diabetes, and appears to be much greater in the young.

Keywords Dementia $\cdot$ Diabetes $\cdot$ Record linkage $\cdot$ Risk $\cdot$ Type $1 \cdot$ Type 2

$\begin{array}{ll}\text { Abbreviations } \\ \text { AD } & \text { Alzheimer's disease } \\ \text { HES } & \text { Hospital Episode Statistics } \\ \text { NHS } & \text { National Health Service } \\ \text { RR } & \text { Rate ratio }\end{array}$

\section{Introduction}

An increasing incidence of type 1 diabetes in children has been documented around the world, and rates are projected to increase further [1-5]. At the population level, the increasing incidence will lead to a higher prevalence of adults with type 1 diabetes in the future, and consequently a higher rate of complications associated with type 1 diabetes. While many of these complications are known [6], important clinical questions remain. Specifically, it is not clear whether type 1 diabetes is associated with an increased risk of dementia in the same way as type 2 diabetes [7]; if it is, what is the risk and how does it change with age? Two common types of dementia are of interest: Alzheimer's disease (AD) and vascular dementia.

There has been much research interest in dementia risk in people with diabetes mellitus. The association between the two conditions has been established and quantified [8-10]. However, the overwhelming majority of published studies focused on type 2 diabetes. While the biological connection 
between type 1 diabetes and cognitive dysfunction is well characterised [11-13], according to our literature search there are no published estimates from large-scale studies of the risk of different types of dementia in individuals with type 1 diabetes.

We sought to fill this gap by analysing national hospital statistics for England (population approximately 50 million). Retrospective analysis of administrative health data allowed for the construction of a very large cohort of individuals with type 1 diabetes, which would be difficult to accomplish through other study designs. Furthermore, it allowed for stratification of results by age group and, thus, for tracking how risk changes throughout life. We report our findings by age group, diabetes type and dementia type.

\section{Methods}

\section{Population and data}

We undertook a retrospective cohort analysis using a dataset of English national Hospital Episode Statistics (HES) linked to a dataset of all deaths in England during the period 1 April 1998 to 31 December 2011 [14]. The HES data include clinical, demographic and administrative patient information on all hospital admissions, including day cases (people admitted who do not stay overnight), in all UK National Health Service (NHS) hospitals. The HES data were supplied by the English national Health and Social Care Information Centre. The linked dataset was built by the Oxford record linkage group using, as identifiers, the encrypted NHS number (unique to each individual) and encrypted postcode, date of birth and sex. Mortality data derive from death registration records supplied by the Office for National Statistics, with identifiers encrypted in the same way, and linked into the HES dataset. The file includes deaths registered up to 31 December 2011. The methods used were similar to those described in previous studies [15, 16], and were, briefly, as follows.

A cohort of people with type 1 diabetes was constructed by identifying each person's first recorded episode of day-case care or inpatient admission for type 1 diabetes, coded in any diagnostic field on the discharge abstract, when aged 30 or older, and with no diagnosis of type 2 diabetes on the same record (to increase the accuracy of categorisation of diabetes type). We excluded those under the age of 30 in the principal analyses because the number of dementia cases in this age group was very small and rates had very wide CIs - the study period was not long enough to allow young individuals time to develop dementia. However, we still report data on these people in the results.

The same approach was used for the type 2 diabetes cohort, except that records were excluded if the first record also had a recorded diagnosis of type 1 diabetes. The cohorts will subsequently be referred to, collectively, as the 'diabetes cohorts', for brevity. The ICD-10 codes used were E10 for type 1 diabetes and E11 for type 2 diabetes (www.who.int/classifications/icd/en/).

A reference cohort was constructed by identifying the first admission for each individual with various other medical and surgical conditions and injuries (see footnote in Table 2). This is based on a 'reference group' of conditions that has been used in other studies of disease associations [15, 17]. The standard epidemiological practice was followed for hospital controls: selecting a diverse range of conditions rather than a narrow range (in case the latter are themselves atypical in the associated risk of dementia). We searched the dataset for any subsequent NHS hospital care for, or death from, dementia (the 'outcome' disease) in the diabetes and the reference cohorts. The ICD codes used for all dementia in the ICD-9 (www.icd9data.com/2007/Volume1) and ICD-10 were 290 and 331.0 and F00-F01 and F03 and G30 (ICD-9 was used for deaths in 1999 and 2000 and ICD-10 for deaths from 2001; ICD-10 was used for hospital discharge data throughout the period covered by this study). These codes include AD (290 in ICD-9 and F00 and G30 in ICD-10), vascular dementia (F01) and unspecified dementia (290 in ICD-9 and F03 in ICD-10). We considered dementia cases to be those coded as the principal or a secondary diagnosis on the discharge abstract. We subdivided those with an admission for dementia into those who were specifically recorded as having an admission for AD (331.0 in ICD-9 and F00 and G30 in ICD-10) and those recorded as having an admission for vascular dementia (290.4 in ICD-9 and F01 in ICD-10). People were included in the diabetes or the reference cohorts if they did not have an admission for dementia either before or at the same time as the admission for diabetes or the reference condition, respectively. This was done with the intention of identifying, as best as was possible, the likelihood that diabetes truly preceded dementia. For the same reason, and to reduce the possibility of surveillance bias (the possibility that dementia might be diagnosed early after an admission for diabetes because the person was under clinical observation), we excluded cases of dementia that occurred within 2 years of the first recorded admission for diabetes. The only exception to this was in the analyses of time intervals, where we specifically looked at diabetes cases occurring within 2 years of admission for dementia, in order to give a complete picture of time-interval data from then until 10 or more years after the index admission.

We analysed the data for men and women separately, as well as together, and at different time intervals between first admission for diabetes and first subsequent admission for dementia. In addition, we analysed the data when each diabetes subtype was coded only as the principal reason for admission (i.e. the person was admitted for the principal reason of managing the diabetes itself), rather than as a secondary diagnosis (i.e. was present but the person was admitted principally for a complication of it or for another condition). 
Statistical analysis

In comparing the diabetes cohorts with the reference cohort, we stratified and then standardised by age (in 5-year age groups), sex, calendar year of first recorded admission, region of residence and quintile of patients' Index of Deprivation score (a standard measure of socioeconomic status used in England). Statistical methods are given in detail elsewhere [16]. The first set of calculations compared the cohorts within the individual strata (e.g. within 5-year age groups, single year of hospital admission). The direct method of standardisation was used with the combined diabetes and reference cohort as the standard population. The stratum-specific rates were then applied to the number of people in each stratum within, first, the diabetes cohorts, and then, separately, the reference cohort. This calculation gave the expected number of cases of dementia in each stratum in the diabetes and reference cohorts. The observed and expected numbers within each stratum were then summed to give the totals in the broader age groups shown in the tables and then at all ages.

Results are expressed as rate ratios (RRs) comparing the diabetes cohorts with the reference cohort, using the formula $\left(O^{\text {diab }} / E^{\text {diab }}\right) /\left(O^{\text {ref }} / E^{\text {ref }}\right)$, where $O$ and $E$ are the observed and expected numbers of dementia in the diabetes and reference cohorts, respectively. The calculation of expected values, the RRs, their CIs and $p$ values used standard published statistical methods [18].

Ethical approval for the programme of work linking and analysing routine medical datasets was obtained from the Central and South Bristol Multi-Centre Research Ethics Committee (04/Q2006/176).

\section{Results}

In our dataset, there were 343,062 people with a hospital admission record of type 1 diabetes mellitus between 1998 and 2011 in England, and 1,855,141 people with type 2 diabetes mellitus (Table 1).

The sex distribution was similar across both diabetes types, with men representing just over a half of all cases. The ratio of controls to cases varied by age but, as the analyses were all done within age strata, this is immaterial. For example, the risk of dementia in the 32,018 people in the diabetes type 1 age group aged 30-39 was compared with the risk in 864,486 $(32,018 \times 27)$ people in the reference cohort; and the risk in the 554,157 people with type 2 diabetes aged 70-79 was compared with around 550,000 people in the same age group in the reference cohort (Table 1). We examined the RRs for dementia in those with diabetes compared with those without diabetes by age and over time. Table 2 shows the adjusted RRs for different types of dementia by type of diabetes and age group. Table 3 illustrates how the overall RRs for different types of dementia changed over time by type of diabetes. The summary of results below is organised by type of diabetes.
Type 1 diabetes

Age at admission There was a significantly elevated overall risk of dementia in people with type 1 diabetes (RR 1.65; $95 \%$ CI 1.61, 1.68) (Table 2). The RR was highest in people admitted to hospital with type 1 diabetes when aged 30-39 $(7.10 ; 4.65,10.6)$ and gradually decreased as age increased, with a much lower RR for those aged 80 or older $(1.16 ; 1.11$, 1.20). There was a small but significant elevated overall risk of AD (RR 1.10; 1.05, 1.15). Similarly, the risk was greatest in those aged 30-39 and reduced with increasing age. For those aged 80 or older, the risk was no longer statistically significant; in fact, there was a reduced risk of $\mathrm{AD}$ in this age group $(0.84 ; 0.76,0.92)$.

There was a twofold elevation in overall risk of vascular dementia $(2.21 ; 2.13,2.28)$ (Table 2). The risk of vascular dementia reduced with increasing age from $14.0(5.04,36.5)$ in those aged $30-39$ to $1.34(1.25,1.45)$ in those aged 80 or older at admission.

Time interval between type 1 diabetes admission and subsequent dementia admission The RR for any dementia was highest when recorded within 2 years of a hospital admission with type 1 diabetes diagnosis $(1.96 ; 1.91,2.02)$ (Table 3$)$; but for the majority of the analyses, we excluded these cases. The RR reduced gradually as the interval between diabetes admission and dementia admission increased, but the overall risk of dementia remained significantly elevated 10 and more years after admission for type 1 diabetes (RR $1.41 ; 1.32,1.51$ ). The results for vascular dementia followed a similar pattern, with the RR decreasing from $2.53(2.41,2.65)$ within the first 2 years of diabetes admission to $1.99(1.78,2.21)$ at 10 or more years after admission. The elevated risk of AD was only seen up to 4 years after diabetes admission.

\section{Type 2 diabetes}

Age at admission There was a significantly elevated overall risk of dementia in people with type 2 diabetes (RR $1.37 ; 1.35$, 1.38) (Table 2). The RR decreased with increasing age from $5.59(3.33,8.99)$ in those aged $30-39$ to $1.10(1.09,1.12)$ in those aged 80 or over. There was no significant elevation in the overall risk of $\mathrm{AD}$ in people with type 2 diabetes, with an $\mathrm{RR}$ of $0.99(0.97,1.01)$. The risk of AD appeared to be meaningfully elevated only for those aged 50-69, raising the possibility that type 2 diabetes may not be a risk factor for $\mathrm{AD}$ in older individuals. Similarly to the individuals with type 1 diabetes, the risk of vascular dementia was significantly elevated in individuals with type 2 diabetes (RR $1.80 ; 1.77,1.83$ ), with the risk decreasing as the age increased.

Time interval between type 2 diabetes admission and subsequent dementia admission The RR for any dementia was highest 
Table 1 Age and sex distribution of the study cohorts: individuals aged 30 or over with a hospital record for type 1 or type 2 diabetes

\begin{tabular}{|c|c|c|c|c|c|c|}
\hline \multirow{2}{*}{$\begin{array}{l}\text { Age at admission } \\
\text { (years) }\end{array}$} & \multicolumn{3}{|l|}{ Type 1 diabetes } & \multicolumn{3}{|l|}{ Type 2 diabetes } \\
\hline & $\begin{array}{l}\text { Exposure cohort, } \\
n \text { (\% of total) }\end{array}$ & $\%$ men & $\begin{array}{l}\text { Ratio of controls } \\
\text { to cases }\end{array}$ & $\begin{array}{l}\text { Exposure cohort, } \\
n \text { (\% of total) }\end{array}$ & $\%$ men & $\begin{array}{l}\text { Ratio of controls } \\
\text { to cases }^{\mathrm{a}}\end{array}$ \\
\hline $30-39$ & $32,018(9.3)$ & 58 & 27 & $38,030(2.1)$ & 56 & 23 \\
\hline $40-49$ & $44,638(13.0)$ & 59 & 21 & $143,570(7.7)$ & 57 & 6 \\
\hline $50-59$ & $58,649(17.1)$ & 58 & 16 & $288,438(15.5)$ & 59 & 3 \\
\hline $60-69$ & $81,740(23.8)$ & 57 & 11 & $468,926(25.3)$ & 58 & 2 \\
\hline $70-79$ & $84,203(24.5)$ & 52 & 9 & $554,157(29.9)$ & 53 & 1 \\
\hline $80+$ & $41,814(12.3)$ & 42 & 11 & $362,002(19.5)$ & 42 & 1 \\
\hline Total & $343,062(100)$ & 55 & 14 & $1,855,141(100)$ & 53 & 2 \\
\hline
\end{tabular}

${ }^{a}$ We included all people eligible to be in the reference cohort: e.g. the risk of dementia in the 32,018 people in the diabetes type 1 age group aged 30-39 was compared with the risk in the 864,486 $(32018 \times 27)$ people in the reference cohort in the same age group. All calculations of observed and expected numbers of 'outcome' conditions were done within each age stratum (in 5-year bands, rather than those showed, for brevity, in the table)

when the admission occurred within 2 years of a hospital admission with type 2 diabetes diagnosis $(1.43 ; 1.41,1.46)$ (Table 3). There was a gradual reduction in the overall dementia risk with increasing time, with the RR remaining significantly elevated 10 or more years after diabetes admission. There was an elevated risk of AD that was only seen within 2 years of diabetes admission. For vascular dementia, the RR was generally stable across all time intervals.

Further sub-analyses We performed a sensitivity analysis by examining the data when diabetes was coded as a principal diagnosis on the record only, rather than a diagnosis found in any position on the record, and found that the resultant RRs were slightly higher than those reported as the main findings of this study (Table 4). The likely explanation is that including only those who were principally admitted to hospital for diabetes, as distinct from having diabetes as a recorded comorbidity, probably means that the study cohort comprised patients with more severe diabetes.

We analysed the data separately for men and women in each age group, but found no appreciable differences (electronic supplementary material [ESM Table 1]).

Finally, we analysed the dataset for dementia in people with diabetes under the age of 30 years within the limits of our period of follow up. Of 69,613 people with type 1 diabetes aged under 30 , there were 3.4 expected cases, 15 observed and an RR of $4.93(2.65,8.54)$; of 17,695 people under 30 with type 2 diabetes, there were 0.7 expected cases, eight observed and an RR of $11.9(4.98,24.4)$.

\section{Discussion}

This study reports RRs for different types of dementia by age group and diabetes type for individuals with a hospital admission record containing a diabetes and/or dementia diagnosis between 1998 and 2011 in England. The results show that type 1 diabetes may be a risk factor for dementia, with an RR for any dementia of 1.65, AD 1.10 and vascular dementia 2.21. Type 2 diabetes was also confirmed as a risk factor for dementia. For both diabetes types, there was a much higher RR in relatively young people that reduced with increasing age.

\section{Type 1 diabetes as a risk factor for dementia}

The findings of this study indicate that type 1 diabetes is associated with an elevated risk of any dementia, $\mathrm{AD}$ and vascular dementia. We cannot compare our findings with those reported in the literature because we were not able to identify any studies that estimated the risk of dementia in a population of individuals with type 1 diabetes. Cardiovascular complications of type 1 diabetes are well established and thus the association of type 1 diabetes with vascular dementia is biologically plausible. It is also known that type 1 diabetes is associated with cognitive impairment $[10,11]$. Therefore, our findings are in line with current understanding of the natural history of type 1 diabetes and the subsequent development of vascular disease.

Two recent meta-analyses of studies on the association of diabetes (both types combined) and dementia [7,9] reported the following relative risks, respectively: $1.51(1.31,1.74)$ and $1.73(1.65,1.82)$ for any dementia; $1.46(1.20,1.77)$ and 1.56 $(1.41,1.73)$ for $\mathrm{AD} ; 2.48(2.08,2.96)$ and $2.27(1.94,2.66)$ for vascular dementia. While our estimates for risk of dementia for type 2 diabetes are lower than the overall figures reported in these meta-analyses, they fall within the range of estimates reported by the individual studies included in the meta-analyses. This provides evidence that our RR estimates for type 1 diabetes are reasonable. However, given the lower estimates for type 2 diabetes, the true elevated risk of dementia among 
Table 2 Occurrence of dementia in individuals aged 30 or over with a hospital record for diabetes, and subdivided into 10-year age bands, excluding cases of dementia that occurred within 2 years of diabetes admission

\begin{tabular}{|c|c|c|c|c|c|c|}
\hline \multirow{2}{*}{$\begin{array}{l}\text { Diabetes type, } \\
\text { (ICD-10 code), } \\
\text { age group (years) }\end{array}$} & \multicolumn{2}{|c|}{ Any dementia (F00-F01, F03, G30) } & \multicolumn{2}{|l|}{$\mathrm{AD}(\mathrm{F} 00, \mathrm{G} 30)$} & \multicolumn{2}{|c|}{ Vascular dementia (F01) } \\
\hline & $\begin{array}{l}\text { No. people } \\
\text { with outcome } \\
(N=70,913)\end{array}$ & $\begin{array}{l}\text { Adjusted RR }{ }^{b} \\
(95 \% \mathrm{CI})\end{array}$ & $\begin{array}{l}\text { No. people } \\
\text { with outcome } \\
(N=14,856)\end{array}$ & $\begin{array}{l}\text { Adjusted RR }{ }^{b} \\
(95 \% \mathrm{CI})\end{array}$ & $\begin{array}{l}\text { No. people } \\
\text { with outcome } \\
(N=24,312)\end{array}$ & $\begin{array}{l}\text { Adjusted RR }{ }^{b} \\
(95 \% \mathrm{CI})\end{array}$ \\
\hline $\begin{array}{l}\text { Type } 1 \text { diabetes (E10), } \\
30 \text { and over }\end{array}$ & 10,786 & $1.65(1.61,1.68)^{* * *}$ & 2,113 & $1.10(1.05,1.15)^{* * *}$ & 3,885 & $2.21(2.13,2.28)^{* * *}$ \\
\hline $30-39$ & 33 & $7.10(4.65,10.6)^{* * *}$ & 7 & $6.28(2.32,14.7)^{* * *}$ & 8 & $14.0(5.04,36.5)^{* * *}$ \\
\hline $40-49$ & 114 & $4.40(3.55,5.40)^{* * *}$ & 21 & $2.54(1.53,4.03)^{* *}$ & 42 & $11.6(7.67,17.4)^{* * *}$ \\
\hline $50-59$ & 501 & $4.09(3.70,4.51)^{* * *}$ & 72 & $1.72(1.33,2.20)^{* * *}$ & 213 & $7.26(6.14,8.56)^{* * *}$ \\
\hline $60-69$ & 2,388 & $2.65(2.53,2.77)^{* * *}$ & 471 & $1.52(1.38,1.67)^{* * *}$ & 1,020 & $3.76(3.51,4.04)^{* * *}$ \\
\hline $70-79$ & 4,987 & $1.61(1.57,1.66)^{* * *}$ & 1,059 & $1.08(1.02,1.15)^{* *}$ & 1,863 & $2.09(1.99,2.19)^{* * *}$ \\
\hline 80 and over & 2,763 & $1.16(1.11,1.20)^{* * *}$ & 483 & $0.84(0.76,0.92)^{* *}$ & 739 & $1.34(1.25,1.45)^{* * *}$ \\
\hline $\begin{array}{l}\text { Type } 2 \text { diabetes (E11), } \\
30 \text { and over }\end{array}$ & 60,127 & $1.37(1.35,1.38)^{* * *}$ & 12,743 & $0.99(0.97,1.01)$ & 20,427 & $1.80(1.77,1.83)^{* * *}$ \\
\hline $30-39$ & 21 & $5.59(3.33,8.99)^{* * *}$ & NR & NR & NR & NR \\
\hline $40-49$ & 153 & $2.66(2.20,3.21)^{* * *}$ & 27 & $1.55(0.99,2.34)$ & 58 & $7.90(5.35,11.7)^{* * *}$ \\
\hline $50-59$ & 1,242 & $2.87(2.66,3.09)^{* * *}$ & 244 & $1.62(1.39,1.88)^{* * *}$ & 560 & $5.63(4.93,6.42)^{* * *}$ \\
\hline $60-69$ & 8,313 & $2.12(2.06,2.18)^{* * *}$ & 1,781 & $1.25(1.18,1.33)^{* * *}$ & 3,577 & $3.24(3.08,3.41)^{* * *}$ \\
\hline $70-79$ & 26,892 & $1.44(1.42,1.47)^{* * *}$ & 6,107 & $1.00(0.97,1.03)$ & 9,832 & $1.84(1.79,1.90)^{* * *}$ \\
\hline 80 and over & 23,506 & $1.10(1.09,1.12)^{* * *}$ & 4,580 & $0.87(0.84,0.91)^{* * *}$ & 6,396 & $1.29(1.25,1.33)^{* * *}$ \\
\hline
\end{tabular}

Data suppressed where there were fewer than five observed cases

${ }^{a}$ Number of people with the outcome disease in the diabetes cohort

${ }^{b}$ Adjusted for sex, age in 5-year bands, time period in single calendar years, region of residence and deprivation score associated with patients' area of residence, in quintiles. RRs were calculated as the ratio of the observed/expected numbers of dementia in the cohort for diabetes, to the observed/ expected numbers in the reference cohort. Conditions used in reference cohort, with Office of Population, Censuses and Surveys (OPCS) code edition 3 for operations and ICD-10 code for diagnosis (with equivalent codes used for other coding editions): appendicectomy (OPCS3 441), hip replacement (810), knee replacement (812), tonsillectomy (230-236), squint (ICD-10 H49-H51), otitis externa, otitis media (H60-H67), varicose veins (I83), haemorrhoids (I84), upper respiratory tract infections (J00-J06), deflected septum, nasal polyp (J33+J34.2), impacted tooth and other disorders of teeth (K00-K03), inguinal hernia (K40), ingrowing toenail and other diseases of nail (L60), sebaceous cyst (L72.1), internal derangement of knee (M23), bunion (M20.1), selected fractures (S42, S52, S62, S82, S92) dislocations, sprains and strains (S03, S13, S23, S33, S43, S53, S63, S73, S83, S93), superficial injury and contusion (S00, S10, S20, S30, S40, S50, S60, S70, S80, S90)

$* * p<0.01$ and $* * * p<0.001$

No., number

$\mathrm{NR}$, not reported

individuals with type 1 diabetes is likely to be even higher than is reported here. Given the limitations of the methods (discussed below), and the fact that associations between type 1 diabetes and vascular dementia were stronger and more clearly present at long time intervals than those with $\mathrm{AD}$, we consider that the evidence is stronger for vascular dementia than for $\mathrm{AD}$.

Published reports on the association between type 2 diabetes and $\mathrm{AD}$ are inconsistent. Our estimate of risk of $\mathrm{AD}$ in individuals with type 2 diabetes (RR $0.99,0.97,1.01$ ) suggests there is no association between the two conditions. It is worth noting that in most of the studies included in recent systematic evaluations of published literature, the CIs for AD risk include $1.0[7,9,19]$.

The understanding of the biological mechanisms that underpin the association between diabetes and dementia is incomplete. Both are complex conditions influenced by a variety of environmental, genetic and biological factors, as well as the interaction of those factors [20]. A number of possible mechanisms that are potentially involved with cognitive dysfunction have been identified for both types of diabetes [12, 13]. In addition, the degree of cognitive impairment has been shown to depend on the age of diabetes onset, and thus its duration, and how well glucose levels are maintained [11-13]. Duration of diabetes, which is typically longer for those affected with type 1 because they are relatively young at onset, could be a critical factor behind the higher risk of developing dementia among people with type 1 than type 2 diabetes.

\section{Changing risk with age}

We found that the influence of diabetes - both type 1 and type 2 - on the risk of dementia decreases with increasing age. 


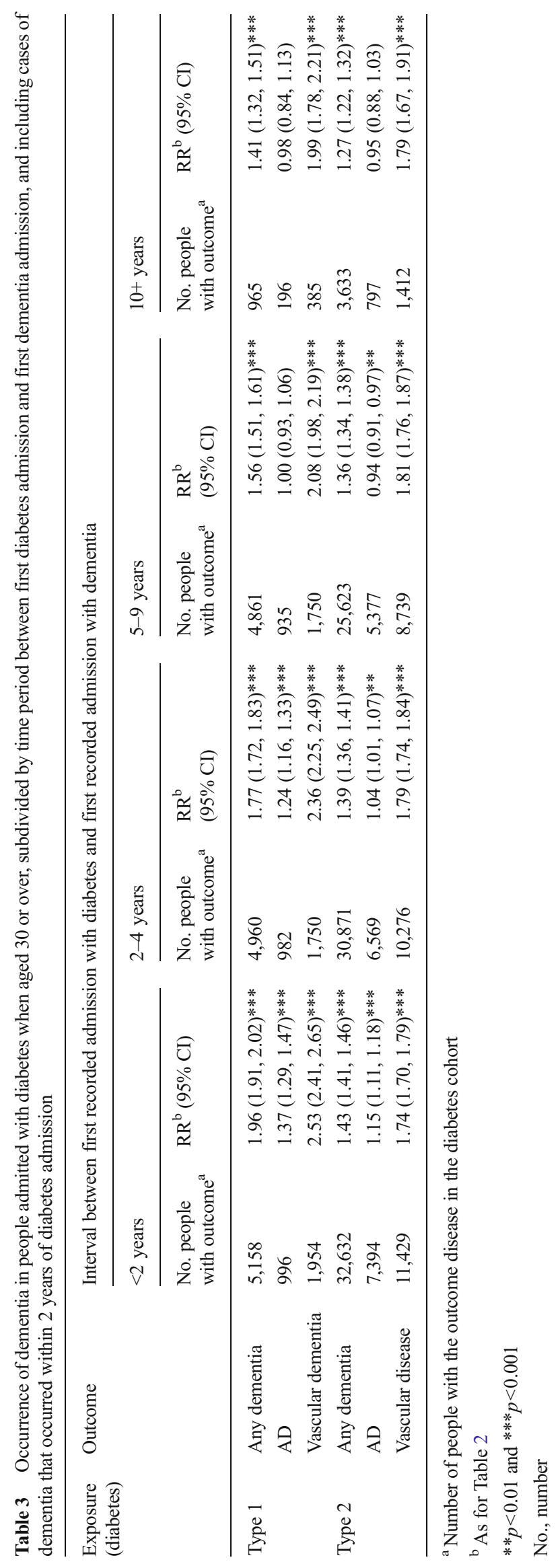


Table 4 Occurrence of dementia in people with diabetes as the principal diagnosis reason for admission

\begin{tabular}{|c|c|c|c|c|c|c|}
\hline \multirow{2}{*}{$\begin{array}{l}\text { Diabetes, type } \\
\text { (ICD code) }\end{array}$} & \multicolumn{2}{|c|}{ Any dementia (F00-F01, F03, G30) } & \multicolumn{2}{|l|}{$\mathrm{AD}(\mathrm{F} 00, \mathrm{G} 30)$} & \multicolumn{2}{|c|}{ Vascular dementia (F01) } \\
\hline & $\begin{array}{l}\text { No. people } \\
\text { with outcome }^{\text {a }}\end{array}$ & $\mathrm{RR}^{\mathrm{b}}(95 \% \mathrm{CI})$ & $\begin{array}{l}\text { No. people } \\
\text { with outcome }^{a}\end{array}$ & $\mathrm{RR}^{\mathrm{b}}(95 \% \mathrm{CI})$ & $\begin{array}{l}\text { No. people } \\
\text { with outcome }\end{array}$ & $\mathrm{RR}^{\mathrm{b}}(95 \% \mathrm{CI})$ \\
\hline Diabetes, type 1 (E10) & 2,382 & $2.12(2.03,2.20)^{* * *}$ & 445 & $1.40(1.27,1.53)^{* * *}$ & 780 & $2.66(2.48,2.86)^{* * *}$ \\
\hline Diabetes, type 2 (E11) & 5,694 & $1.83(1.78,1.88)^{* * *}$ & 1,053 & $1.27(1.19,1.35)^{* * *}$ & 1,701 & $2.28(2.17,2.39)^{* * *}$ \\
\hline
\end{tabular}

\footnotetext{
${ }^{a}$ Number of people with the outcome disease in the diabetes cohort

${ }^{\mathrm{b}}$ As for Table 2

$* * * p<0.001$

No., number
}

Other studies that compared dementia risk by age found that it was lower in individuals aged 65 and over than in individuals younger than 65 [21, 22]. A recent analysis of data from Western Australia that examined survival outcomes in dementia patients, based on history of diabetes (but without specification of type), demonstrated that the impact of diabetes on dementia gradually decreases with increasing age [23]. The study concluded that it is the early onset of diabetes that is accountable for much of the increased risk of dementia in younger age groups, as opposed to a major change in the natural history of diabetes-related dementia.

\section{Sensitivity analysis}

Although our main results were derived from the analysis of records with any mention of diabetes diagnosis, we additionally analysed the data for records where diabetes was the principal reason for admission to hospital. We did this to test the robustness of the results in the absence of other serious comorbid diseases that may actually have been associated with dementia. We found that the RRs were actually slightly higher when using the principal diagnosis of diabetes only, reassuring us that our main reported findings were not due to associations between dementia and other serious comorbid conditions.

\section{Strengths and limitations}

The strengths of our datasets include the large size of the study population, the complete national representation, the inclusion of all ages; and that it comprises recent data. It included over one-third of a million people with type 1 diabetes and nearly two million with type 2 . To the best of our knowledge, this is the first study to report estimates of dementia risk for individuals with type 1 diabetes. Further, we believe that this is also the first study to report age-specific RRs for different types of dementia in patients with different types of diabetes from the same population. The reference cohort has been used extensively in previous studies and has been shown to report plausible rates for a variety of conditions [15-17]. Furthermore, our obtained RR estimates for type 2 diabetes, although lower, are in agreement with the literature, thus lending credibility to our estimates for type 1 diabetes. It is possible that there is some surveillance bias in our study - the identification of cases of dementia soon after a diagnosis of diabetes - but this alone would not explain similarities between our findings at longer follow up and the literature on type 2 diabetes in studies with different designs.

One of the main limitations was the incomplete capture of all cases of diabetes and dementia that occurred in England during the study period, due to the dataset being limited to people admitted to hospital or receiving day case specialist care. As such, absolute rates of dementia among people with diabetes cannot be calculated, but relative rates can. Of the individuals in the reference cohort, $1.1 \%$ subsequently had a record of diabetes and moved to the exposure cohort, i.e. there was a prevalence of hospitalised diabetes of $1.1 \%$ in individuals otherwise eligible to be in the reference cohort. Survey data show that the prevalence of diagnosed diabetes increased in England from $3.3 \%$ to $6.3 \%$ in men, and from $2.5 \%$ to $5.3 \%$ in women, between 1998 and 2010 [24]. Therefore, our prevalence is an underestimate because our dataset does not include people admitted to hospital outside of the study period. A recent study reported that approximately $60 \%$ of patients with type 2 diabetes are hospitalised at least once during a 4 year period [25]. Our study period was substantially longer than that, and we likely captured the majority of people with type 2 diabetes in England.

It is possible that some diabetes-type and dementia-type cases in our data were misdiagnosed. Because of the nature of administrative data and its collection, there will inevitably be some misdiagnosis or miscoding. Unfortunately, privacy regulations preclude independent checking of samples of diagnoses, and their diagnostic basis, in medical records by researchers. It is worth noting that the lack of diagnostic specificity of dementia type affected both cases and controls, and would probably have a limited impact on the RRs comparing the two. We were also not able to control for unrecorded risk factors (e.g. smoking) in our analysis. It is uncertain how this might have affected the findings. 
The RRs in this study, therefore, should be considered indicative rather than definitive and precise. In particular, however, the results strongly suggest that type 1 diabetes, as well as type 2 diabetes, increases the risk of subsequent vascular dementia. The evidence for this is particularly strong in the findings in the relatively young age groups. The RRs for vascular dementia observed among those aged 40-59 (Table 2) were both significantly high in their own right (compared with the reference cohort) and also significantly higher than those for $\mathrm{AD}$ in the corresponding age groups. We suggest that these results point to a real association between type 1 diabetes and vascular dementia because it is unlikely that such high RRs could be a sole product of misclassification of type 2 as type 1 or other uncontrolled confounding factors.

\section{Conclusion}

This study adds new evidence that type 1 diabetes is probably associated with dementia. It also shows how the risk of dementia changes with age, and that the association between diabetes and dementia is particularly strong in people with diabetes when young. Our findings suggest a much stronger association between type 1 diabetes and vascular dementia than with AD. Given that the presented findings are based on hospital statistics alone, further research is required to confirm or refute these associations in the whole population of people with diabetes.

Acknowledgements The linked data sets were built over many years by Leicester Gill and Matt Davidson, Unit of Health-Care Epidemiology, University of Oxford, Oxford, UK.

Funding The Unit of Health-Care Epidemiology was funded by the English National Institute for Health Research to build the linked dataset. The views expressed in this paper do not necessarily reflect those of the funding body. K. Smolina is funded in part by the Banting Postdoctoral Fellowship from the Canadian Institutes for Health Research. M. Goldacre is funded in part by Public Health England.

Duality of interest The authors declare that there is no duality of interest associated with this manuscript.

Contribution statement All authors conceived the study. MJG designed the study. CJW analysed the data. CJW and KS drafted the paper. All authors interpreted the data, reviewed the paper and approved the final version. MJG is the guarantor of this work.

\section{References}

1. Patterson CC, Gyurus E, Rosenbauer J et al (2012) Trends in childhood type 1 diabetes incidence in Europe during 1989-2008: evidence of non-uniformity over time in rates of increase. Diabetologia 55:2142-2147
2. Vehik K, Hamman RF, Lezotte D et al (2007) Increasing incidence of type 1 diabetes in 0 - to 17 -year-old Colorado youth. Diabetes Care 30:503-509

3. DIAMOND Project Group (2006) Incidence and trends of childhood type 1 diabetes worldwide 1990-1999. Diab Med J Br Diab Assoc 23: 857

4. Tran F, Stone M, Huang CY et al (2014) Population-based incidence of diabetes in Australian youth aged 10-18 yr: increase in type 1 diabetes but not type 2 diabetes. Pediatr Diabetes 15:585590

5. Patterson CC, Dahlquist GG, Gyurus E, Green A, Soltesz G (2009) Incidence trends for childhood type 1 diabetes in Europe during 1989-2003 and predicted new cases 2005-20: a multicentre prospective registration study. Lancet 373:2027-2033

6. Atkinson MA, Eisenbarth GS, Michels AW (2014) Type 1 diabetes. Lancet 383:69-82

7. Gudala K, Bansal D, Schifano F, Bhansali A (2013) Diabetes mellitus and risk of dementia: a meta-analysis of prospective observational studies. J Diab Investig 4:640-650

8. Biessels GJ, Staekenborg S, Brunner E, Brayne C, Scheltens P (2006) Risk of dementia in diabetes mellitus: a systematic review. Lancet Neurol 5:64-74

9. Cheng G, Huang C, Deng H, Wang H (2012) Diabetes as a risk factor for dementia and mild cognitive impairment: a meta-analysis of longitudinal studies. Intern Med J 42:484-491

10. Cukierman T, Gerstein H, Williamson J (2005) Cognitive decline and dementia in diabetes - systematic overview of prospective observational studies. Diabetologia 48:2460-2469

11. Brands AM, Biessels GJ, De Haan EHF, Kapelle LJ, Kessels RPC (2005) The effects of type 1 diabetes on cognitive performance: a meta-analysis. Diabetes Care 28:726-735

12. McCrimmon RJ, Ryan CM, Frier BM (2012) Diabetes and cognitive dysfunction. Lancet 379:2291-2299

13. Sims-Robinson C, Kim B, Rosko A, Feldman EL (2010) How does diabetes accelerate Alzheimer disease pathology? Nat Rev Neurol 6: 551-559

14. Gill L, Goldacre M (2003) English national record linkage of hospital episode statistics and death registration records. Report to the Department of Health, 2003. Oxford, Unit of Health-Care Epidemiology. Available from: http://nchod.uhce.ox.ac.uk/ NCHOD $\% 20$ Oxford $\% 20$ E $5 \% 20$ Report $\% 201$ st $\% 20$ Feb VerAM2. pdf, accessed 1 Feb 2015

15. Seminog OO, Goldacre MJ (2013) Risk of pneumonia and pneumococcal disease in people with severe mental illness: English record linkage studies. Thorax 68:171-176

16. Gold J, Goldacre R, Maruszak H, Giovannoni G, Yeates D, Goldacre M (2014) HIV and lower risk of multiple sclerosis: beginning to unravel a mystery using a record-linked database study. J Neurol Neurosurg Psychiatry 86:9-12

17. Wotton CJ, Yeates DG, Goldacre MJ (2011) Cancer in patients admitted to hospital with diabetes mellitus aged 30 years and over: record linkage studies. Diabetologia 54:527-534

18. Breslow N, Day N (1987) Statistical methods in cancer research, volume II. The design and analysis of cohort studies. IARC scientific publication No. 82. 1987, Lyon: International Agency for Research in Cancer. 103-115

19. Kopf D, Frölich L (2009) Risk of incident Alzheimer's disease in diabetic patients: a systematic review of prospective trials. J Alzheimers Dis 16:677-685

20. Strachan MW, Mark MJ, Reynolds RM, Marioni RE, Price JF (2011) Cognitive function, dementia and type 2 diabetes mellitus in the elderly. Nat Rev Endocrinol 7:108-114

21. Cheng P-Y, Sy H-N, Wu S-L, Wang W-F, Chen Y-Y (2012) Newly diagnosed type 2 diabetes and risk of dementia: a population-based 7-year follow-up study in Taiwan. J Diabetes Complicat 26:382-387 
22. Xu W, Qiu C, Gatz M, Pederson NL, Johansson B, Fratiglioni L (2009) Mid- and late-life diabetes in relation to the risk of dementia a population-based twin study. Diabetes 58:71-77

23. Zilkens RR, Davis WA, Spilsbury K, Semmens JB, Bruce DG (2013) Earlier age of dementia onset and shorter survival times in dementia patients with diabetes. Am J Epidemiol 177:1246-1254

24. British Heart Foundation (2010) Prevalence of diagnosed diabetes by sex and country, UK 1991-2010, Table 5.32. Available from: www. bhf.org.uk/research/heart-statistics/risk-factors/diabetes.aspx, accessed 1 Feb 2015

25. Khalid JM, Raluy-Callado M, Curtis BH, Boye KS, Maguire A, Reaney M (2014) Rates and risk of hospitalisation among patients with type 2 diabetes: retrospective cohort study using the UK general practice research database linked to English Hospital Episode Statistics. Int J Clin Pract 68:4048 